\title{
MicroRNA profiling of breast cancer tissue using an LNA-based microarray
}

\begin{abstract}
Abnormal expression of microRNAs (miRNAs) in cancer suggests their applicability as diagnostic and prognostic signatures. Here we describe a new microarray platform, miRCURYTM LNA, based on Locked Nucleic $A c i d\left(L N A^{T M}\right)$-modified capture probes that have uniquely high affinities for miRNAs. We applied this platform to study the global expression profiles of miRNAs in breast cancer with the aim of identifying potential new biomarkers.
\end{abstract}

miRNAs are short 21-26-nucleotide noncoding RNA molecules that can regulate the expression of genes involved in many biological processes, including development, differentiation and apoptosis ${ }^{1}$. Approximately half of all human miRNA genes are localized in fragile chromosomal regions that are associated with various human cancers ${ }^{2}$. Additionally, several miRNAs, referred to as 'oncomirs', can have a direct role in oncogenesis by acting as tumor suppressors and oncogenes ${ }^{3}$. That miRNAs comprise a new class of promising diagnostic and prognostic biomarkers has been suggested in a recent study showing that a molecular signature consisting of about 200 miRNAs was superior for classification of cancer to mRNA expression profiles containing more than 20,000 genes ${ }^{4}$.

Today, global gene expression analysis based on microarray technology has facilitated a new molecular taxonomy for classification of cancer. In particular, for diagnosis of breast cancer, several gene signatures have been reported that allow stratification of patients in, for example, a good and a poor prognosis group ${ }^{5}$. Such gene signatures, however, have not yet entered clinical practice ${ }^{6}$, which suggests the need for even better and more accurate molecular tumor markers; for example, ones based on miRNA profiles.

The miRCURY LNA Array platform contains capture probes for all registered and annotated miRNAs from the miRBase. Additionally, the platform contains several miRPlus ${ }^{\mathrm{TM}}$ capture probes for miRNAs not yet annotated in the miRBase. Thus, the miRCURY LNA Array platform has a strong discovery potential and is very well suited for generation of miRNA molecular signatures.

\section{LNA-enhanced miRNA profiling}

The small size of miRNAs poses a unique challenge in their detection, requiring a method that is both highly sensitive and discriminatory ${ }^{7}$.

Thomas Litman, Nana Jacobsen, Mikkel Nørholm, Christian Glue, Nina Stahlberg \& Søren Møller

Exiqon A/S, Bygstubben 9, DK-2950 Vedbaek, Denmark. Correspondence should be addressed to T.L. (support@exiqon.com).
Most commercially available technologies for global miRNA expression profiling are based on DNA microarrays, in which the probes are spotted onto a glass surface. Such probes, however, are not optimal for miRNA detection because of cross-hybridization and lack of specificity; discrimination between single-nucleotide differences is not always possible ${ }^{8}$.

LNAs are a class of conformationally restricted nucleotide analogs. The incorporation of LNA in an oligonucleotide increases the affinity of that oligonucleotide for its complementary RNA or DNA target, increasing the melting temperature $\left(T_{\mathrm{m}}\right)$ of the duplex. Additionally, the $T_{\mathrm{m}}$ difference between a perfectly matched target and a mismatched target is substantially higher than that observed when a DNA-based oligonucleotide is used. These properties- high $T_{\mathrm{m}}$ and excellent mismatch discrimination-make LNA-modified probes ideal for analysis of short

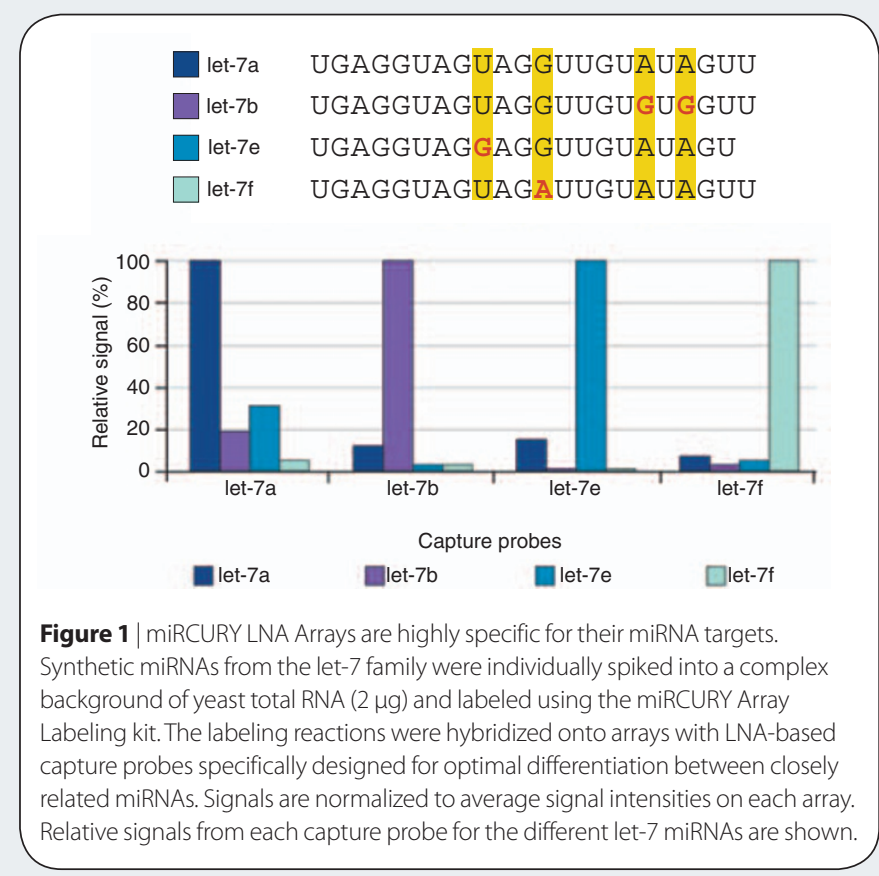




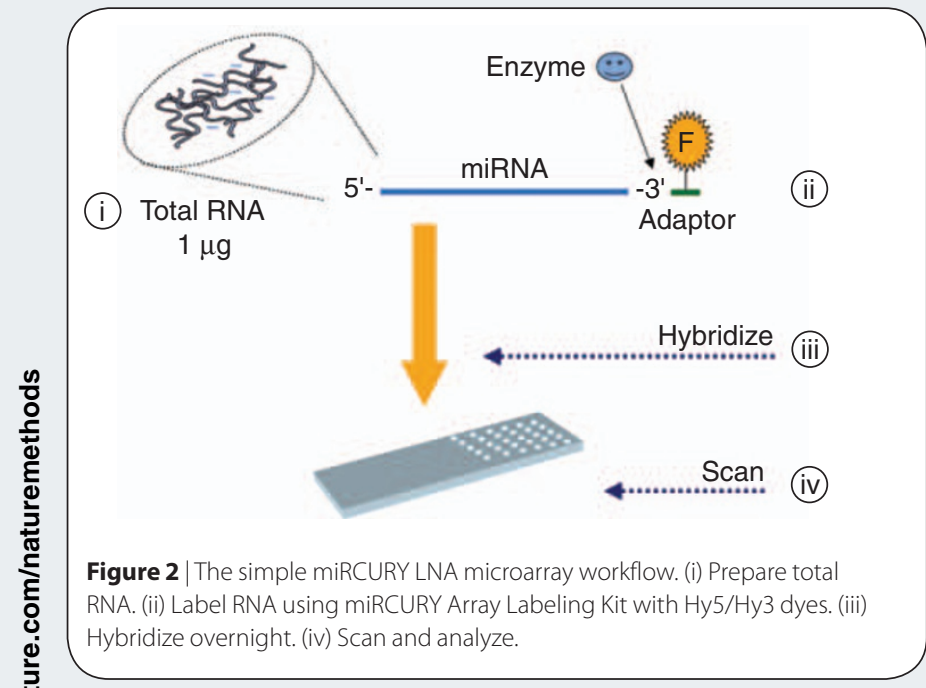

and very similar targets like miRNAs (Fig. 1). Furthermore, by adjusting the LNA content and probe length, it is possible to design $T_{\mathrm{m}}$-normalized probes, thereby allowing hybridization conditions that are optimal for all microarray capture probes. Moreover, the high sensitivity of LNAmodified capture probes eliminates the need for miRNA enrichment of the sample, allowing the use of very small amounts of total RNA directly for hybridization and reducing the possibility of losing miRNAs during sample preparation.

\section{Simple labeling and hybridization procedure}

We applied the miRCURY LNA microarray platform to generate miRNA expression profiles of breast cancer tissue and normal adjacent breast tissue to identify possible new biomarkers for breast cancer ${ }^{9}$.

After RNA isolation from the breast tissue by phenol-chloroform extraction, we labeled $1 \mu \mathrm{g}$ total RNA in a fast and simple one-step procedure using the miRCURY Array Labeling kit. Then we analyzed the RNA for miRNA expression on miRCURY LNA Arrays containing $T_{\mathrm{m}}$-normalized capture probes for 1,488 miRNAs, which include all known miRNAs as well as 151 proprietary human miRPlus sequences not yet annotated in miRBase. The labeling and hybridization procedure is outlined in Figure 2.

\section{Specific and robust data}

Our analysis of miRNAs in breast cancer and normal tissue (Fig. 3) revealed many differentially expressed miRNAs, including those reported earlier to be associated with breast cancer, such as several members of the let-7 family and miR-21 (ref. 10). We also identified several miRNAs that have not previously been connected with breast cancer. Some of these miRNAs may represent new molecular signatures with diagnostic and prognostic promise for patients with breast cancer. We are presently validating the new potential miRNA biomarkers with LNA-enhanced real-time PCR assays.

\section{Conclusion}

LNA-enhanced miRCURY microarrays have superior sensitivity, excellent specificity, and high intra-array and interbatch reproduci-

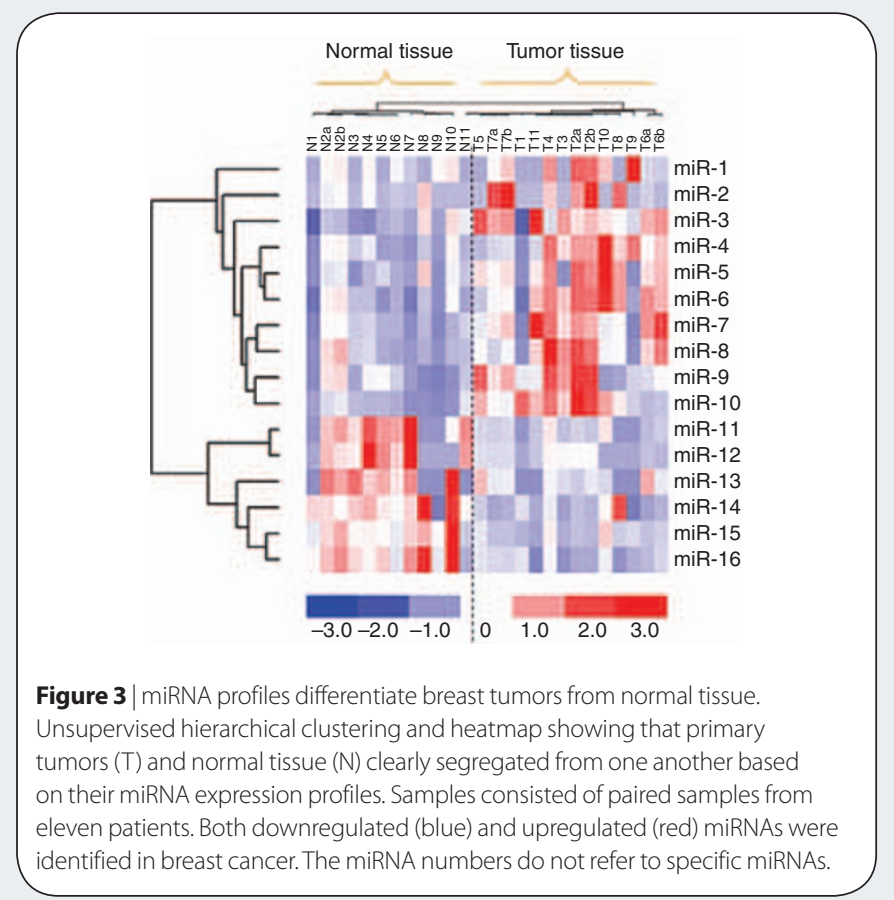

bility. The fast and simple RNA labeling procedure eliminates the need for amplification or miRNA enrichment, which minimizes RNA handling that could otherwise potentially introduce experimental bias.

By applying the miRCURY LNA microarray platform to normal and cancerous tissues, we were able to identify miRNA biomarkers for breast cancer. To learn more about the miRCURY LNA Array products and our microRNA Profiling Services, please visit http:// www.exiqon.com/array or http://www.exiqon.com/services.

\section{ACKNOWLEDGMENTS}

We thank the MAMBIO Group at Herlev University Hospital for kindly providing the tissues included in Figure 3.

1. Miska, E.A. How microRNAs control cell division, differentiation and death. Curr. Opin. Genet. Dev. 15, 563-568 (2005).

2. Calin, G. A. et al. Human microRNA genes are frequently located at fragile sites and genomic regions involved in cancers. Proc. Natl. Acad. Sci. USA 101, 29993004 (2004).

3. Esquela-Kerscher, A. \& Slack, F. J. Oncomirs-microRNAs with a role in cancer. Nat. Rev. Cancer 6, 259-269 (2006).

4. Lu, J.et al. MicroRNA expression profiles classify human cancers. Nature $\mathbf{4 3 5}$, 834-838 (2005).

5. van de Vijver, M.J. et al. A gene-expression signature as a predictor of survival in breast cancer. N. Engl. J. Med. 347, 1999-2009 (2002).

6. Loi, S. et al. Molecular forecasting of breast cancer: time to move forward with clinical testing. J. Clin. Oncol. 24, 721-722 (2006).

7. Krützfeldt, J., Poy, M.N. \& Stoffel, M. Strategies to determine the biological function of microRNAs. Nat. Genet. 38, S14 (2006).

8. Baskerville, S. \& Bartel, D.P. Microarray profiling of microRNAs reveals frequent coexpression with neighboring miRNAs and host genes. RNA 11, 241-247 (2005).

9. Castoldi, M. et al. A sensitive array for microRNA expression profiling (miChip) based on locked nucleic acids (LNA). RNA 12, 913-920 (2006).

10. Iorio, M.V. et al. MicroRNA gene expression deregulation in human breast cancer. Cancer Res. 65, 7065-7070 (2005).

This article was submitted to Nature Methods by a commercial organization and has not been peer reviewed. Nature Methods takes no responsibility for the accuracy or otherwise of the information provided. 